indicate fewer medical attendances, fewer treated respiratory chest infections, and improved overall weight gain in the screened population. We did not find significantly fewer hospital admissions. The number of days in hospital, which have been variously reported as reduced ${ }^{6}$ or increased, ${ }^{5}$ were not analysed. All of our screened patients were briefly admitted on diagnosis for assessment and education about their condition. The later age of diagnosis and possible lesser awareness of the importance of recognising symptoms before diagnosis in the unscreened group might have accounted for the similar admission rates in the first two years of life.

As yet there have been no adequate studies of the psychosocial implications of neonatal screening for cystic fibrosis. The potential effects of the resampling and false postive results ${ }^{5}$ and the effects of diagnosing the disease in 'well' babies have to be considered against the observed findings in this study of repeated medical consultations and delay in diagnosis in a sick child; there is also the advantage with earlier diagnosis of genetic counselling. The psychosocial disadvantages of screening are becoming less obvious now that there is a reduced need for resampling and an increase in the predictive value of a positive result (resulting from more specific assays). There is also increasing experience with the approach to diagnosis in 'well' babies, and conversely the avoidance of delayed diagnosis now that fetal genetic diagnosis is possible.

In conclusion, the published results of screening for cystic fibrosis indicate that screening pro- grammes deserve wider application and study. ${ }^{1} 46$ In particular, as both repeated pulmonary infections and relative underweight are important adverse prognostic indicators in cystic fibrosis, a reduced morbidity should be regarded as one of the primary advantages of screening.

We are grateful to the staff of the cystic fibrosis clinics. Royal Children`s and Mater Children's Hospitals; to the Cystic Fibrosis Association of Queensland, especially Drs I Robertson. RM Harris, and Mr E Ryan for providing access to patient records; and to the families of cystic fibrosis patients who provided information.

\section{References}

' Crossley JR, Smith PA. Edgar BW, Gluckman PD, Elliott RB. Neonatal screening for cystic fibrosis using immunoreactive trypsin assay in dried blood spots. Clin Chim Acta 1981;113: $111-21$.

2 Bowling FG. Walson AR, Rylatt DB. Elliott JE, Bunch RS Bundersen PG. A monoclonal antibody based trypsinogen EIA for screcning of neonates for cystic fibrosis. Lancet 1987;i:826-7.

${ }^{3}$ Bowling FG, Brown ARD. Newborn screening for cystic fibrosis using an ELISA technique. Clin Chem Acta 1987 (in press).

+ Wilcken B, Brown ARD, Urwin R, Brown DA. Cystic fibrosis screening by dried blood spot trypsin assay: results in 75000 newborn infants. J Pediatr 1983;102:383-7.

5 Ad Hoc Committec Task Force on neonatal screening, Cystic Fibrosis Foundation. Pediatrics 1983:72:741-5.

${ }^{6}$ Wilcken B, Chalmers G. Reduced morbidity in patients with cystic fibrosis detected by neonatal screening. Lancet 1986:ii: 1319-21.

Correspondence to Dr F Bowling, Neonatal Screening Unit. State Department of Health, 63 George Street, GPO Box 495, Brisbane. Qucensland, Australia 4001.

Received 4 August 1987

\title{
Cognitive development in transposition of the great vessels
}

\author{
N HESZ AND E B CLARK \\ Institute of Child Health, Westminster and Charing Cross Medical School and Department of Pediatrics, \\ Division of Developmental Disabilities, University of Iowa, Iowa City, USA
}

SUMmaRY Ten children who had had transposition of the great vessels (TGV) repaired, deep hypothermia, and cardiac arrest were examined. Seven children with acyanotic heart disease and 12 unaffected siblings were tested for comparison. Their intelligence, academic achievement, and behaviour was studied. The group with TGV had lower performance subscores on the intelligence test, an increase in somatic complaints, and aggressive behaviour.
Survival of children with transposition of the great vessels (TGV) improved substantially with the advent of balloon atrial septostomy ${ }^{1}$ and intracardiac baffle. ${ }^{2}$ Children with TGV may have associated hemiplegia due to cerebrovascular accidents ${ }^{3}$ or a dyskinetic movement disorder due to anaesthetic hypothermia and cardiac arrest ${ }^{4}$ or delayed development related to restricted cardiac reserve and chronic hypothermia and parental imposition of restrictions of the child's activities. The severe neurological damage some children have made us question whether seemingly unaffected 
children may exhibit subtle neurological and intellectual deficits.

\section{Subjects and methods}

Thirty three children with TGV were treated at the University of Iowa between 1967 and 1984. Four children died. Of the 29 survivors, 14 met the following criteria: age 6.5 to 14 years, no perinatal asphyxia, gestation of $>37$ weeks, and no associations of mental handicap-for example, Down's syndrome. Two families were untraceable, one family had moved, and one family chose not to participate. A total of 10 children $(71 \%$ of those eligible) were tested.

Eight children had a Rashkind balloon atrial septostomy followed by the insertion of a Mustard baffle before 2 years of age; this included one child who had a Blalock Hanlon procedure at 6.5 months, followed by a Mustard operation at 29 months. One child had a Waterton shunt at 13 months and a Rastelli procedure at 6.5 months, followed by a Mustard operation at 29 months. Ten operations, one in each child, were performed under deep hypothermia and cardiac arrest. Postoperative complications included one complex arrhythmia that required a pacemaker. Two children suffered a cardiac arrest and were immediately resuscitated. One child required dopamine for circulatory support in the immediate postoperative period. No child had a cerebrovascular accident or convulsion. Two children were thought to have a residual heart disease, and one required revision of his Mustard baffle during the initial period in hospital to repair superior vena cava and pulmonary vein obstructions. One child had late superior vena cava obstruction requiring balloon angioplasty then a vein graft. In six children the postoperative recovery was uncomplicated.

For comparison, children with ventricular septal defect were selected by criteria identical with those for children with TGV. Of the 14 children who met these criteria, two had moved and one had uncontrolled fits and was mentally handicapped. Three families chose not to participate, therefore eight children were tested (57\% of those eligible). All had had a transatrial repair of the defect: two children had deep hypothermia and cardiac arrest; the others were on cardiac bypass for an average of 50 minutes.

Postoperative complications included one child who had a residual ventricular septal defect that required a second operation and one child who had atrioventricular dissociation after surgery that required medical management. None of these children had had cerebrovascular accidents, convulsions, or cardiac arrest.

Seven siblings of children with TGV and five siblings of the children with ventricular septal defect were tested for comparison.

A psychologist who was blind to the design and intent of the study tested all children. He administered the (revised) Weschler intelligence scale for children, a continuous performance task providing an objective measure of fine motor skill, and the Wide Range Achievement Test (a normed reading, spelling, and arithmetic test). Parents completed an Achenback behaviour scale, which is a comprehensive checklist for common behavioural problems in childhood, for which normative data are available by age and sex. A paediatrician interviewed the family and collected developmental milestones from their baby books and school records and performed a general and neurological examination on each child.

The results were analysed by Student's $t$ test for making comparisons with population norms. The two groups of children with heart disease were also compared with their siblings using the $t$ test for independent samples and for comparing the mean of

Table 1 Mean subscores for IQ from children with transposition of great vessels and three comparison groups of children

\begin{tabular}{|c|c|c|c|c|c|c|}
\hline & \multicolumn{6}{|c|}{ Intelligence quotient } \\
\hline & \multicolumn{2}{|c|}{ Verbal } & \multicolumn{2}{|c|}{ Performance } & \multicolumn{2}{|c|}{ Full scale } \\
\hline & (mean & $(S D))$ & (mean & $(S D))$ & (mean & $(S D))$ \\
\hline \multirow{4}{*}{$\begin{array}{l}\text { Children with transposition of the great vessels } \\
(n=10) \\
\text { Children with ventricular septal defect } \\
(n=8) \\
\text { Siblings of children with transposition of the great vessels } \\
(n=7) \\
\text { Siblings of children with ventricular septal defect } \\
(n=5)\end{array}$} & $93 \cdot 0$ & $(14 \cdot 28)$ & $92 \cdot 0$ & $(9 \cdot 26)$ & $91 \cdot 6$ & $(11 \cdot 98)$ \\
\hline & 99.9 & $(8 \cdot 75)$ & $99 \cdot 4$ & $(11 \cdot 6)$ & $99 \cdot 1$ & $(9 \cdot 82)$ \\
\hline & $95 \cdot 4$ & $(10 \cdot 56)$ & $98 \cdot 0$ & $(17 \cdot 48)$ & $96 \cdot 4$ & $(14 \cdot 42)$ \\
\hline & $102 \cdot 6$ & $(5 \cdot 03)$ & $98 \cdot 8$ & $(12 \cdot 80)$ & $100 \cdot 6$ & $(9 \cdot 10)$ \\
\hline
\end{tabular}


Table 2 Academic achievement test subscores of children with transposition of the great vessels and three comparison groups of children

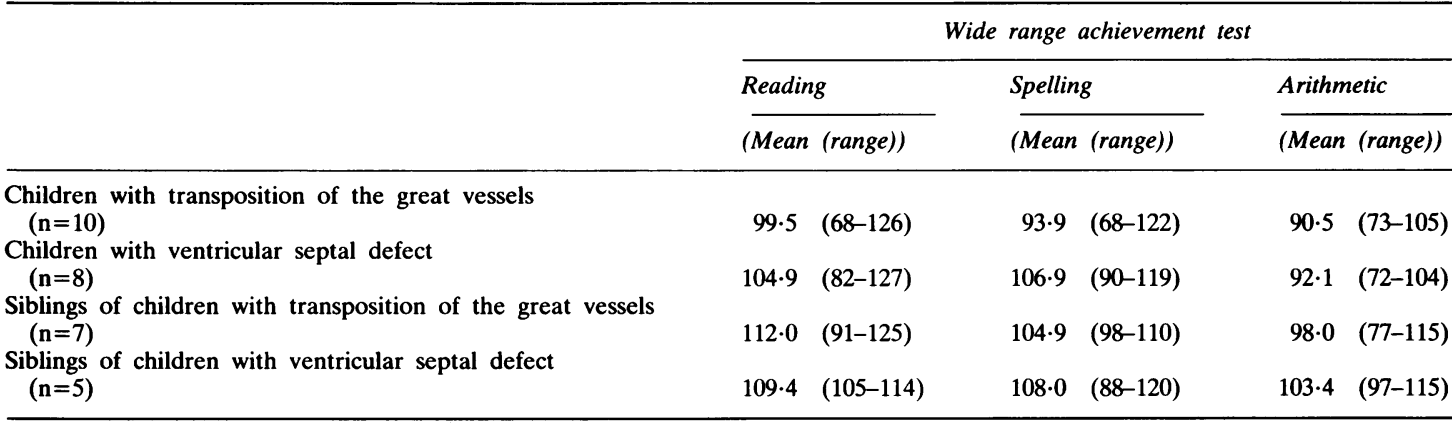

the difference between each child-sibling pair. The medical ethics committee of the University of Iowa approved the study.

\section{Results}

The mean intelligence quotients of all children are shown in table 1 . The only significant differences from the general population were for the children with TGV where three verbal subtests (vocabulary, arithmetic, and comprehension) and three performance subtests (digit span, block designs, and coding) were all below population norm $(p<0 \cdot 05)$. The mean difference of the sibling pairs' scores showed a significant difference between the group with TGV $v$ sibling pairs and ventricular septal defect $v$ sibling pairs on performance IQ $(p<0.03)$.

The results of the achievement test are shown in table 2 . In comparing achievement subscore differences between sibling pairs there was a consistent trend for both groups of children with heart disease to have lower scores than their siblings. For the continuous performance task there were no differences between sibling pairs. On the Achenbach behaviour checklist, the children with TGV were more aggressive $(p<0.02)$ and had more somatic complaints $(p<0.3)$ than did children with a ventricular septal defect. No neurological abnormalities were found for any child. There were no differences in ages at which standard milestones were reached.

\section{Discussion}

Our data suggest that children with transposition of the great vessels do have greater impairment of cognitive skills than do children with ventricular septal defect, although in both diseases there is a similar degree of deprivation of stimulation and potential for exploration because of debility in infancy. These children with TGV did not have impaired academic achievement to a degree equal to the impairment of performance IQ but increase in somatic complaints and aggressive behaviour suggested subtle problems with their peers at school. Interestingly, a degree of social imperceptiveness seems to accompany visual perceptual problems in other populations of children with poor performance IQ and learning disabilities. ${ }^{6}$

With advances in the diagnosis of developmental disorders and advances in educational and remedial treatment of learning disorders it seems timely to begin identifying more specifically the developmental disorder that is associated with a specific disease.

\section{References}

${ }^{1}$ Rashkind WJ. Palliative procedures for transposition of the great arteries. Br Heart J 1971;39(suppl 1):69.

2 Mustard WT, Keith JD, Trusler GA, Fowler R, Kidd L. Surgical management of transposition of the great vessels. J Thorac Cardiovas Surg 1964;48:953.

3 Cottril CM, Kaplan S. Cerebral vascular accidents in cyanotic congenital heart disease. Am J Dis Child 1973;125:484-7.

4 Brunberg JA, Reilly EL, Doty DB. Central nervous system consequences in infants of cardiac surgery using deep hypothermia and circulatory arrest. Circulation 1974;49-50(suppl II):60-8.

${ }^{5}$ Newburger JW, Silbert AR, Buckley LP, et al. Cognitive function and age at repair of transposition of the great arteries in children. N Engl J Med 1984;310:1495-9.

${ }^{6}$ Pickar DB. Psychosocial aspects of learning disabilities. Bulletin of the Menninger Clinic 1986;50:22-32.

Correspondence to Dr N Hesz, Department of Child Health, Charing Cross and Westminster Medical School, Charing Cross Hospital, Fulham Palace Road, London W6 8RF.

Received 5 August 1987 